Acta Crystallographica Section C

Crystal Structure

Communications

ISSN 0108-2701

\title{
Ethyl N-Methyl-2-pyrrolylcarbonylhydrazinocarboxylate
}

M. Liu-Gonzalez, F. Sanz-Ruiz, M. E. Gonzalez-Rosende, O. Lozano-Lucía, E. Zaballos-García and J. Sepulveda-Arqués 


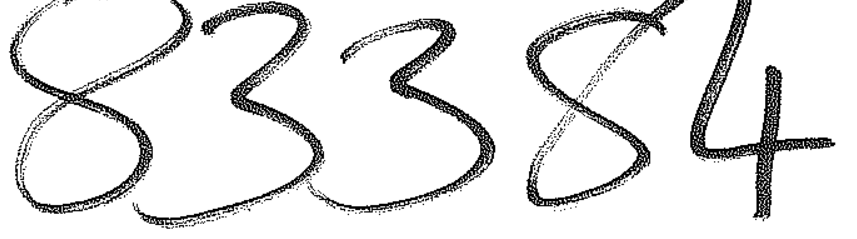

KIII

Values of lo*fobs and lok

Page 1

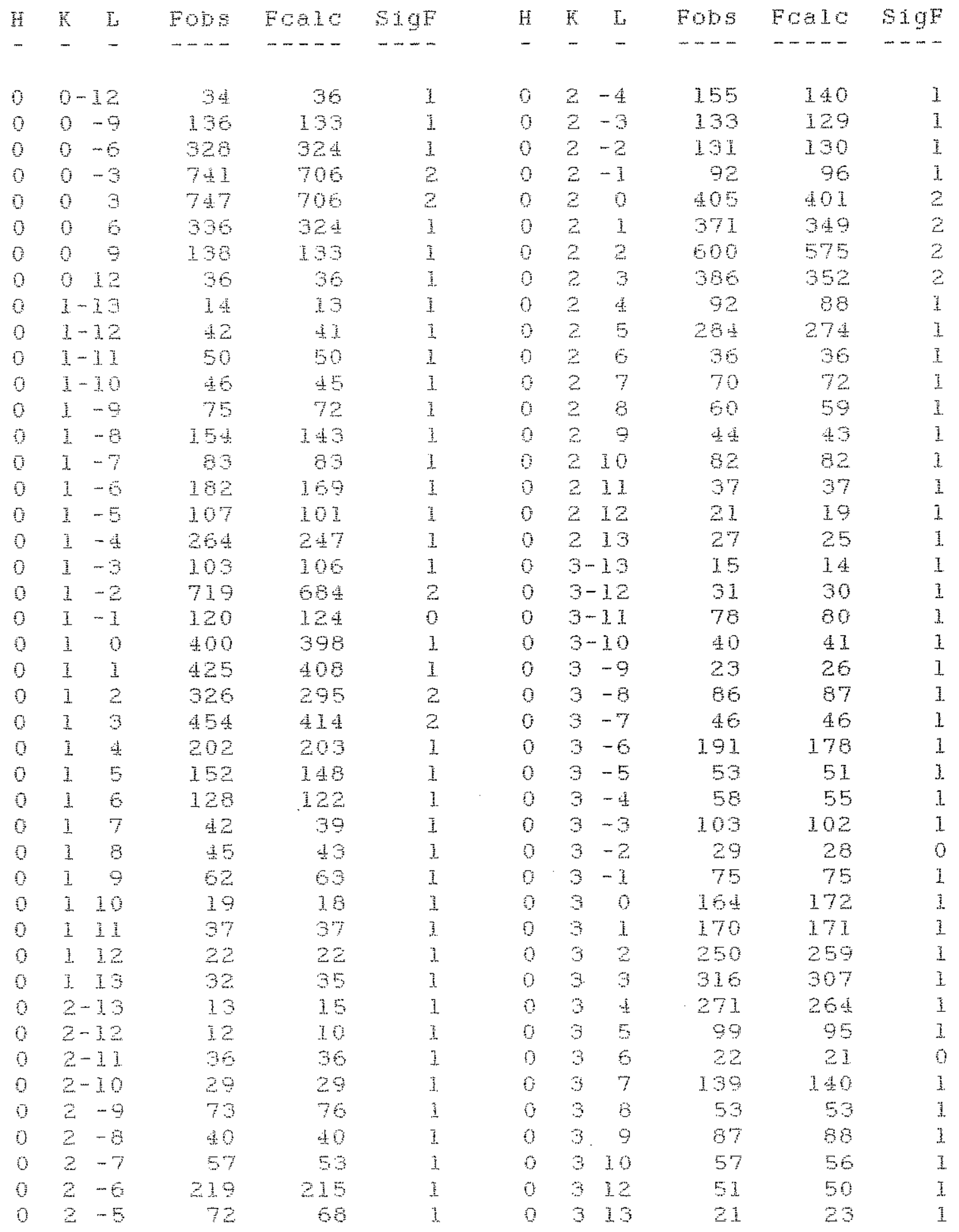




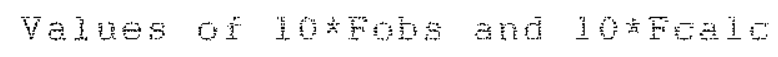

page 2

\begin{tabular}{|c|c|c|c|c|c|c|c|c|c|c|c|}
\hline Ex & 型 & $i=$ & Fols & $F c a 10$ & 819 & II & In & $\bar{t}$ & mobs & 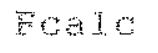 & $19 F$ \\
\hline - & - & - & $--\cdots$ & $\cdots-\cdots$ & $\cdots-\cdots$ & - & - & - & $\cdots-\cdots$ & $-\cdots-\cdots$ & $-\cdots$ \\
\hline 0 & & 10 & 11 & 3 & 1 & 0 & 5 & 10 & 48 & 4 & .1 \\
\hline 0 & 3 & -9 & 55 & 59 & 1 & 0 & 5 & 12 & 27 & 28 & 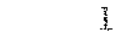 \\
\hline 0 & 4 & -8 & 40 & $\frac{d i}{2}$ & 1 & 0 & & 12 & 28 & 28 & 1 \\
\hline 0 & 4 & -7 & 86 & 87 & 1. & 0 & $6-$ & 10 & 27 & 27 & 1. \\
\hline 0 & 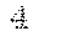 & -6 & 27 & 27 & 0 & 0 & 6 & -9 & 38 & 38 & I \\
\hline 0 & 4 & -5 & 56 & 54 & 1 & 0 & 6 & -8 & 23 & 23 & I \\
\hline 0 & 4 & -4 & 52 & 53 & 1. & 0 & 6 & -6 & 20 & 21 & 1 \\
\hline 0 & 4 & -3 & 75 & 74 & 1 & 0 & 6 & -5 & 43 & 45 & 1 \\
\hline 0 & $d$ & -2 & 153 & 351 & $\mathrm{I}$ & 0 & 6 & - & 31 & 31 & 0 \\
\hline 0 & $\frac{4}{3}$ & -1 & 307 & 308 & 1 & 0 & 6 & -3 & 70 & 71 & 1 \\
\hline 0 & 4 & 0 & 44 & $4 E$ & 1 & 0 & 6 & -2 & 43 & 45 & 1 \\
\hline 0 & 4 & 1 & 260 & 257 & 1 & 0 & 6 & -1 & 45 & 45 & 1 \\
\hline 3 & 4 & 2 & 285 & 280 & 1 & 0 & 6 & 0 & 58 & 57 & I \\
\hline 0 & 4 & 3 & 94 & 96 & $\frac{1}{1}$ & 0 & 6 & 1 & 22 & 22 & 0 \\
\hline 0 & 4 & 4 & 46 & 49 & 1 & 0 & 6 & 2 & 20 & 19 & 0 \\
\hline 0 & 4 & 5 & 263 & 259 & 1 & 0 & 6 & 3 & 41 & 38 & 1 \\
\hline 0 & 4 & 6 & 52 & 49 & $I$ & 0 & 6 & 4 & 60 & 68 & 3 \\
\hline 0 & 4 & 7 & 124 & 123 & 1 & 0 & 6 & 5 & 4 & 4 & 1 \\
\hline 0 & 4 & 8 & 120 & 127 & I & 0 & 6 & 6 & 32 & 32 & I \\
\hline 0 & 4 & 9 & 53 & 52 & 1 & 0 & 6 & 7 & 33 & 35 & I \\
\hline 0 & & 10 & 94 & 96 & 金 & 0 & 6 & 9 & 54 & 57 & l. \\
\hline 0 & & 13 & 18 & 16 & 1 & 0 & 6 & 11 & 20 & 18 & 1 \\
\hline 0 & $5-$ & 12 & 30 & $3 \pi$ & 1. & 0 & $7-$ & 12 & 21 & $2 I$ & 1 \\
\hline 0 & $5-$ & 11 & $3 \pm$ & 35 & 1 & 0 & 7 & -9 & 25 & 26 & 1 \\
\hline 0 & $5-$ & 10 & 20 & 27 & 1 & D & 7 & -8 & 16 & 18 & 3 \\
\hline 9 & 5 & -9 & 45 & 47 & $\mathrm{j}$ & 0 & 7 & -7 & 36 & 36 & ]. \\
\hline 0 & 5 & -6 & 17 & 18 & 1 & 0 & 7 & -6 & 85 & 85 & 1 \\
\hline 0 & 5 & -7 & 50 & 51 & 1 & 0 & 7 & -5 & 98 & 100 & 1 \\
\hline 0 & 5 & -6 & 1.45 & 150 & 1 & 0 & 7 & -4 & 30 & 30 & 1 \\
\hline 0 & 5 & -5 & 9 & 7 & 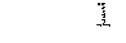 & 0 & 7 & -3 & 75 & 76 & 1 \\
\hline 0 & 5 & -4 & 8 & 79 & 1 & 0 & 7 & -2 & 75 & 76 & 1 \\
\hline 0 & 5 & -3 & 125 & 130 & $i$ & 0 & 7 & -1 & 47 & 49 & 1 \\
\hline 0 & 6 & -2 & 21 & $2 i$ & 0 & 0 & 7 & 0 & 26 & 28 & 0 \\
\hline 0 & 5 & -1 & 72 & 73 & 1 & 0 & 7 & 1 & 37 & 37 & I. \\
\hline 0 & 5 & 0 & 25 & 27 & 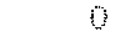 & 0 & 7 & 2 & 38 & 41 & 1 \\
\hline 0 & 5 & 1 & 166 & 165 & 1 & 0 & 7 & 3 & 44 & 47 & 1 \\
\hline 0 & 5 & 2 & 58 & 57 & 1 & 0 & 7 & 4 & 93 & 97 & I \\
\hline 0 & 5 & 3 & 61 & 64 & 1 & 0 & 7 & 5 & 26 & 27 & 1 \\
\hline 0 & 5 & 4 & 59 & 59 & 1. & 0 & 7 & 6 & 31 & 31 & 1 \\
\hline 0 & 5 & 5 & 59 & 66 & 1. & 0 & 7 & 7 & 27 & 28 & 1 \\
\hline 0 & 5 & 6 & 16 & 16 & 1 & 0 & 7 & 9 & 24 & 24 & 1 \\
\hline 0 & 5 & 7 & 30 & 29 & 1 & 0 & 8 & -9 & 13 & 12 & 1 \\
\hline t & 5 & 8 & 55 & 58 & $I$ & 0 & 8 & -7 & 9 & 9 & I \\
\hline 0 & 5 & 9 & 59 & 50 & 1 & 0 & 8 & -6 & 17 & 16 & 1 \\
\hline
\end{tabular}




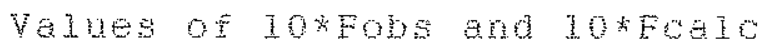

Page 3

\begin{tabular}{|c|c|c|c|c|c|c|c|c|c|c|}
\hline H & 蓇 & Fobs & Fondo & $8+9$ & $\ddot{H}$ & $\mathrm{~K}$ & $I_{1}$ & Fobs & $\operatorname{Fac} \mathrm{C}$ & $51 \mathrm{gF}$ \\
\hline- & - & $-\cdots-$ & ----- & ---- & - & - & - & ---- & $-\cdots-\cdots$ & ---- \\
\hline 0 & -5 & 36 & 35 & $\underline{z}$ & 3 & 0 & -9 & 62 & 62 & $I$ \\
\hline 0 & -4 & 34 & 36 & I. & 1 & 0 & -8 & 46 & 43 & 1 \\
\hline 0 & -3 & 43 & 42 & I & 1 & 0 & -7 & 42 & 40 & 1 \\
\hline 0 & -2 & 30 & 30 & 1 & 1 & 0 & -6 & 128 & 122 & 1 \\
\hline 0 & 8 & 14 & 1 & 1 & 1 & 0 & -5 & 153 & 149 & $I$ \\
\hline 0 & $B$ & 13 & 13 & 1. & 1 & 0 & -4 & 201 & 203 & 1 \\
\hline 0 & 8 & 21 & 22 & 1 & 1 & 0 & -3 & 458 & 41 & 2 \\
\hline 0 & 8 & $\frac{40}{10}$ & 4.4 & $I$ & $I$ & 0 & -2 & 325 & 295 & 2 \\
\hline 0 & 8 & 29 & 27 & 1 & 3 & 0 & -1 & 429 & 408 & 1 \\
\hline o & 8 & 12 & 12 & 1 & 1. & 0 & 0 & 405 & 398 & 1 \\
\hline 0 & $\theta$ & 5 & 9 & $I$ & $i$ & 0 & $\mathrm{I}$ & 121 & 124 & $\mathrm{O}$ \\
\hline 0 & 9 & 30 & 28 & 1 & 1 & 0 & 2 & 726 & 684 & 2 \\
\hline 0 & -7 & 24 & 24 & 1 & 1 & 0 & 3 & 100 & $10 \%$ & 1 \\
\hline 0 & -6 & 3 & 32 & 1 & 1 & 0 & 4 & 264 & 247 & 1 \\
\hline 0 & -5 & 25 & 24 & 1. & 1. & 0 & 5 & 105 & 101 & i \\
\hline 0 & -4 & 20 & 20 & 1 & 1 & 0 & 6 & 584 & 3.69 & 1 \\
\hline 3 & -3 & 39 & 30 & ] & 1 & 0 & 7 & 81 & 83 & 1 \\
\hline 0 & -1 & 12 & 11 & $I$ & 1 & 0 & 8 & 152 & 143 & 1 \\
\hline 0 & 0 & 31 & 30 & 1 & 1 & 5 & 9 & 75 & 72 & 1 \\
\hline 0 & 1 & 1.3 & 13 & 1 & 1 & 0 & 10 & 46 & 45 & $I$ \\
\hline 0 & 9 & 9 & 8 & $I$ & 1 & 0 & 1 & 49 & 50 & 1 \\
\hline 0 & 9 & 10 & 19 & 1 & 1 & 0 & 12 & 42 & 41 & 1 \\
\hline 0 & 9 & 20 & 19 & 1 & $I$ & 0 & 13 & 10 & 13 & 3 \\
\hline 0 & 9 & 19 & 18 & 1 & 1 & & 11 & 70 & 72 & 1 \\
\hline 0 & 9 & 11 & 10 & I & $\lambda$ & & 10 & 34 & 35 & 1 \\
\hline 0 & 10 & 13 & I I & 1 & I & 1. & -9 & 27 & 27 & $I$ \\
\hline 0 & 10 & 24 & 24 & 3. & $I$ & 1 & -8 & $6 I$ & 60 & 1 \\
\hline 0 & 10 & 15 & 13 & 1 & 1 & 1 & -7 & 160 & 153 & 1 \\
\hline 0 & 10 & 27 & 26 & 1 & 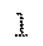 & $I$ & -6 & $8 I$ & 73 & 1 \\
\hline 0 & 10 & 19 & 17 & 1 & ]. & 1. & -5 & 50 & 52 & 1 \\
\hline 0 & 10 & 15 & 17 & 1 & $I$ & I & -4 & 162 & 150 & 1 \\
\hline 0 & 10 & 17 & 15 & $\underline{i}$ & 1 & 1 & -3 & 229 & 226 & 1. \\
\hline 0 & 10 & 19 & 17 & $I$ & $I$ & $\mathcal{I}$ & -2 & 732 & 700 & 2 \\
\hline 0 & 10 & $2 \mathrm{I}$ & 19 & $I$ & $i$ & 1 & -1 & 566 & 563 & 2 \\
\hline 0 & 1 & 16 & 盖的 & 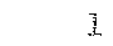 & 1 & 1 & 0 & 5,17 & 507 & 2 \\
\hline 0 & 1.1 & 24 & 23 & 1 & 1 & 1 & 1 & 148 & 152 & 0 \\
\hline 0 & $1 \mathrm{I}$ & 17 & 3 & 1 & 1 & 1 & 2 & 44 & 410 & 2 \\
\hline 0 & 11 & $2 i$ & 19 & 1 & $I$ & 1 & 3 & 261 & 256 & I \\
\hline 0 & 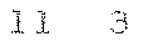 & 26 & 23 & 1 & 1 & 1 & 푬 & 16 & 17 & 0 \\
\hline 0 & 114 & 20 & 37 & 1 & 1 & 1 & 5 & 62 & 52 & $\underline{1}$ \\
\hline$\underline{I}$ & $0-13$ & 35 & 35 & I. & 1 & 1 & 6 & 58 & 57 & 1 \\
\hline 1 & $0-12$ & $2+\frac{1}{2}$ & 2 & 1 & 1 & 1 & 7 & 111 & $10 \%$ & 1 \\
\hline 1 & $0-11$ & 3 & 37 & 1 & 1 & I. & 8 & 39 & 30 & 1 \\
\hline 1 & $0-10$ & 20 & 10 & 1. & 1 & 1 & 9 & 12 & 1 & 3. \\
\hline
\end{tabular}




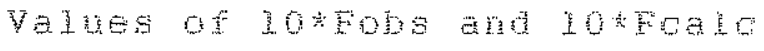

page 4

\begin{tabular}{|c|c|c|c|c|c|c|c|c|c|c|}
\hline H & 1 & $\mathrm{Eot} \mathrm{s}$ & Fed $1=$ & $\Leftrightarrow 19 F$ & $\mathrm{H}$ & I & $I$ & $\mathrm{Bas}$ & FGa & $919 \mathrm{~F}$ \\
\hline- & - & ---- & $-\cdots \cdots$ & ---- & - & - & - & $\ldots-\cdots$ & $-\cdots--$ & $-\cdots$ \\
\hline$I$ & 11 主 & 52 & 50 & 3 & 1 & 3 & 4 & 14 & 139 & 1. \\
\hline$i$ & 12 & 5 & 13 & 1 & 3 & 3 & $F_{3}$ & 92 & 95 & $\underline{I}$ \\
\hline 1 & 113 & 50 & 24 & 3 & $i$ & 3 & 6 & 50 & 53 & 1 \\
\hline 1 & $2-13$ & 23 & 25 & 1 & 1 & 3 & 7 & 95 & 92 & 1 \\
\hline 1 & $2-12$ & 59 & 57 & 1 & $\underline{I}$ & 3 & 8 & 47 & 147 & 1 \\
\hline I & $2-11$ & 25 & 26 & 1 & 1 & 3 & 9 & 04 & 88 & 1 \\
\hline 1 & $2-30$ & 50 & 47 & 战 & 1 & 3 & 10 & 54 & 53 & 1. \\
\hline 1 & $2-9$ & 35 & 31 & $I$ & 1 & 3 & 12 & 38 & 41 & $I$ \\
\hline$\underline{I}$ & $2-8$ & 15 & 0 & 1. & $I$ & et & 12 & 27 & 6 & 1 \\
\hline 1 & $2-7$ & 26 & 25 & 0 & $I$ & & 11 & 50 & 50 & 1 \\
\hline 1 & -6 & $20 \div$ & .96 & I & 1 & & 10 & .11 & 9 & $I$ \\
\hline 1 & -5 & 30 & 27 & 0 & 1 & 生 & -9 & 14 & 13 & l. \\
\hline 3 & - & 121 & 114 & 1 & $I$ & 4 & -8 & 62 & 66 & $I$ \\
\hline 1 & -3 & 95 & 87 & 1 & 1 & 4 & -7 & 59 & 62 & 1 \\
\hline 1 & -2 & 172 & $17 \%$ & $\mathrm{I}$ & $I$ & 4 & -6 & 80 & 81 & I \\
\hline 1 & $2-1$ & 1.32 & 163 & 1 & 1 & 4 & -5 & 9 & 10 & $I$ \\
\hline 1 & 20 & 147 & 156 & $J$ & $\frac{1}{2}$ & 4 & -4 & 52 & 47 & 1 \\
\hline 1 & 1 & 192 & 196 & 1 & 1 & 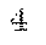 & -3 & 21 & 20 & 0 \\
\hline 1 & 2 & 377 & 345 & 2 & 1 & 4 & -2 & 119 & 117 & I \\
\hline i & 3 & 315 & 317 & 1 & 1 & 4 & -1 & 194 & 191 & 1 \\
\hline 1 & 4 & 127 & 120 & 1 & 1 & 4 & 0 & 82 & 81 & 1 \\
\hline 1 & 5 & 236 & 228 & 1 & 1 & 4 & 1. & 248 & 248 & 1 \\
\hline 1 & $\mathrm{E}$ & $5 \%$ & 54 & L & $\underline{1}$ & 4 & 2 & 25 & 23 & 0 \\
\hline$\underline{1}$ & 7 & 25 & 22 & 0 & 1 & $d$ & 3 & 156 & 158 & 3. \\
\hline 1 & 8 & Af & 19 & 1 & 3 & 4 & 4 & 120 & 112 & $\underline{1}$ \\
\hline 1 & 9 & 73 & 71 & 1 & 1 & 4 & 5 & 154 & 359 & 1 \\
\hline 1 & 10 & 47 & 47 & I. & 1 & 4 & 6 & 79 & 85 & $\mathrm{~L}$ \\
\hline 1 & 211 & 21 & 20 & 1 & 3 & $\frac{9}{7}$ & 7 & 166 & 171 & 1 \\
\hline$\tilde{I}$ & 212 & 32 & 32 & i & 3 & 4 & 8 & 39 & 41 & 1 \\
\hline 3 & $3-12$ & 39 & 31 & 1 & 3 & 4 & 9 & 4 & 44 & I \\
\hline$\underline{I}$ & $3-11$ & 35 & 30 & 1 & $i$ & 1 & 10 & 85 & 85 & 1 \\
\hline 1 & $7 \quad-9$ & 90 & 97 & 1 & 1 & i & 11 & 11 & 15 & 1 \\
\hline 3 & $3-3$ & 78 & 39 & 0 & i. & & 12 & 24 & 25 & 1 \\
\hline 1 & $3-7$ & 5 & 50 & 3 & 1 & & 11 & 21 & 21 & 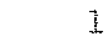 \\
\hline$\underline{z}$ & $3-6$ & 100 & 93 & 1 & $I$ & 5 & 10 & 25 & 26 & 3 \\
\hline l & -5 & 37 & 31 & 1 & 1 & 5 & -9 & 71 & 75 & I \\
\hline 1 & $3-4$ & 299 & 292 & $I$ & 1 & 5 & -8 & 33 & 32 & 1 \\
\hline I & $3-3$ & 97 & 9 & $\mathrm{I}$ & 1 & 5 & -7 & 33 & 34 & I \\
\hline 1 & $3-2$ & 79 & 75 & $I$ & $\bar{z}$ & 5 & -6 & 89 & 92 & $I$ \\
\hline 1 & $3-1$ & 162 & 170 & 1 & 1 & 5 & -5 & $3 i$ & 33 & 0 \\
\hline 1 & 30 & 176 & 180 & 3 & 1 & 5 & -4 & 25 & 23 & 0 \\
\hline 1 & 1 & 254 & 261 & 1 & 1 & 5 & -3 & 85 & 84 & 1 \\
\hline 1 & 2 & 206 & 206 & 1 & 1 & 5 & -2 & 29 & 28 & 0 \\
\hline 1 & 33 & 222 & 220 & 1 & $\mathrm{I}$ & 5 & -1 & 140 & 140 & I \\
\hline
\end{tabular}




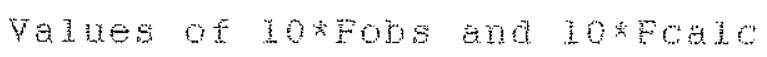

Page 5

\begin{tabular}{|c|c|c|c|c|c|c|c|c|c|c|c|}
\hline$H$ & E & $\tilde{I}_{i, i}$ & 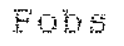 & 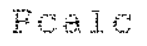 & $31 \mathrm{~F}$ & $\mathrm{H}$ & 茫 & $I_{1}$ & Fobs & $F G a I 6$ & $S j g F$ \\
\hline- & - & - & ---- & $-\cdots-\cdots$ & $-\cdots$ & - & - & - & $-\cdots$ & ---- & $-\ldots$ \\
\hline 1 & 5 & 0 & 65 & 66 & $I$ & 1 & 7 & 1 & 66 & 70 & $I$ \\
\hline 1 & 5 & 1 & 105 & 106 & 1. & 1 & 7 & 2 & 30 & 31 & 1 \\
\hline 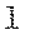 & 5 & 2 & 75 & 74 & 3 & 1 & 7 & 3 & 52 & 57 & 1 \\
\hline 1 & 5 & 3 & 78 & 78 & 1 & $I$ & 7 & 4 & 52 & 57 & 1 \\
\hline 1 & 5 & $\underline{\dot{a}}$ & 50 & 57 & 1 & 1 & 7 & 5 & 20 & $2 I$ & 1 \\
\hline 1. & 5 & 5 & 34 & 35 & 0 & 1 & 7 & 6 & 17 & 17 & $I$ \\
\hline 1 & 5 & 6 & 26 & 25 & 0 & 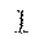 & 7 & 7 & 35 & 34 & 1 \\
\hline I & 5 & 7 & 20 & 21 & 1 & 1 & 7 & 8 & 23 & 26 & 1 \\
\hline$\frac{3}{2}$ & 5 & $\theta$ & 47 & 47 & $\bar{J}$ & 1 & 7 & 9 & 31 & 28 & 1 \\
\hline 1 & 5 & 9 & 5.5 & 56 & 1 & 2 & 7 & 11 & 11 & 9 & 2 \\
\hline 1 & 5 & 11 & 28 & 29 & 1 & 1 & & -10 & $I 6$ & 1. 4 & 1 \\
\hline 1 & 5 & 12 & 19 & 20 & 3 & 1 & 8 & -5 & 15 & 14 & 1 \\
\hline I & $\theta$ & 12 & 24 & 24 & 1 & 1 & 8 & -7 & 30 & 30 & I. \\
\hline 1 & 6 & -9 & 23 & 25 & 1 & 3 & 8 & -6 & 30 & 30 & 1 \\
\hline 1 & $b$ & -8 & 53 & 53 & i. & 1 & $\beta$ & -5 & 23 & 1.3 & 1 \\
\hline I & 6 & -7 & $=9$ & 50 & $i$ & .1 & 8 & -4 & 51 & 51 & 1 \\
\hline 1 & 6 & -6 & 53 & 57 & 1 & 1 & 8 & -3 & 49 & 51 & 1 \\
\hline 1 & $\vec{b}$ & -5 & 29 & 30 & 1 & 1 & 8 & -2 & 50 & 50 & ] \\
\hline i & 5 & -4 & 25 & 25 & 1 & 1 & 8 & -1 & 38 & 17 & $I$ \\
\hline $\bar{l}$ & 6 & -3 & 71 & 76 & 1 & I & 8 & 0 & 31 & 30 & 1 \\
\hline 1 & $B$ & -2 & 4 & $\Delta$ & 1 & 1 & 8 & 7 & 28 & 29 & I \\
\hline 1 & 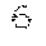 & -1 & 36 & 37 & 0 & $I$ & 8 & 2 & 25 & 25 & $I$ \\
\hline 1 & $\varepsilon$ & 0 & $\frac{3}{-1} 4$ & 45 & $\underline{E}$ & in & 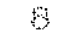 & 9 & 36 & $3 \mathrm{~B}^{2}$ & 1 \\
\hline 1 & $b$ & 1 & 79 & 81 & $I$ & $\mathrm{I}$ & $\beta$ & 4 & 10 & 17 & 1. \\
\hline$I$ & 6 & 3 & 70 & 74 & I & $\bar{I}$ & 8 & 5 & 30 & 30 & 1 \\
\hline I. & 5 & 4 & 15 & 17 & 1 & 1 & 8 & 6 & 13 & 10 & 3 \\
\hline 1 & 6 & 5 & 40 & 42 & $\tilde{I}$ & 1 & 8 & 7 & $\times 5$ & 14 & 1 \\
\hline 1 & 6 & 6 & 37 & 39 & 1 & 1 & 8 & $\beta$ & 9 & 7 & 1 \\
\hline 1 & 6 & 7 & 39 & 42 & $i$ & 1 & 8 & 9 & 26 & 25 & 1 \\
\hline 1 & 6 & 8 & 19 & 18 & $\vec{j}$ & $I_{n}$ & 9 & -8 & 12 & ]. 1 & I \\
\hline 1 & 5 & 9 & 19 & 16 & $I$ & $I$ & 9 & -7 & 23 & 22 & 1 \\
\hline 1 & 6 & 10 & 31 & 31 & 1 & 1 & 9 & -6 & 23 & 21 & 1 \\
\hline 1 & 6 & 11 & 22 & 20 & 1 & 1 & 9 & -5 & 36 & 34 & 1 \\
\hline 1 & 7 & 10 & 13 & 12 & 1 & 1 & 9 & $-\frac{1}{1}$ & 21 & 22 & 1 \\
\hline I & 7 & -9 & 23 & 22 & $\underline{1}$ & 1 & 9 & -3 & 16 & 15 & 1 \\
\hline 3 & 7 & -8 & 12 & 13 & $\mathrm{I}$ & 1 & 9 & -2 & 11 & 10 & 3. \\
\hline 1 & 7 & -7 & 28 & 27 & 1 & $\mathfrak{I}$ & 9 & $-I$ & s. & 39 & 1 \\
\hline 1 & 7 & -6 & 11 & 40 & 1 & 1 & 9 & 0 & 17 & 14 & 1 \\
\hline 1 & 7 & -5 & 66 & 67 & $I$ & 1 & 9 & 3 & 24 & 23 & I. \\
\hline 1 & 7 & $-\frac{3}{4}$ & 55 & 56 & I & $I$ & 9 & 5 & 1.4 & 13 & 1 \\
\hline 1 & 7 & -3 & 51 & 50 & 1 & 1 & 9 & $b$ & 1.3 & 15 & 1 \\
\hline $\bar{I}$ & 7 & -2 & 73 & 71 & $z$ & I & 30 & -3 & 12 & 12 & 1 \\
\hline 1 & 7 & -1 & 55 & 56 & $I$ & 1 & 10 & 1 & 13 & 13 & 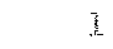 \\
\hline 1 & 7 & 0 & 81 & $3 \pm$ & 0 & $!$ & 10 & 9 & 22 & 20 & 1 \\
\hline
\end{tabular}




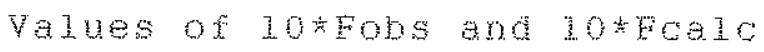

Page 6

\begin{tabular}{|c|c|c|c|c|c|c|c|c|c|}
\hline$\dot{H i}$ & Is & $\mathrm{Fols}$ & Fealc & $\operatorname{sig}$ & F & $\mathrm{I}_{\mathrm{s}}$ & Fobs & Foalc & $S 1 g F$ \\
\hline - & - & $-\cdots$ & $-\cdots$ & $\ldots \ldots$ & - & - & $-\cdots$ & ---- & $-\cdots$ \\
\hline 1 & 104 & 3 & 12 & 1 & 2 & 3 & 165 & 268 & I \\
\hline 2 & $0-13$ & 25 & 25 & ] & 2 & 4 & 105 & 103 & 1 \\
\hline 2 & $0-12$ & 21 & 19 & 1 & 2 & 5 & 105 & 102 & $j$ \\
\hline 2 & $0-11$ & 38 & 37 & $\lambda$ & 2 & 6 & 109 & 107 & 1 \\
\hline 2 & $0-10$ & 84 & 82 & 1 & 2 & 7 & 25 & 24 & 0 \\
\hline 2 & $0-9$ & 4 & 13 & 1 & 2 & 8 & 21 & 22 & 1 \\
\hline 2 & $0-8$ & 60 & 59 & 1 & 2 & 9 & 55 & 55 & 1 \\
\hline 2 & $0-7$ & 70 & 72 & 1. & 2 & $1 \quad 10$ & 23 & 22 & 1. \\
\hline 2 & $0-6$ & 37 & 36 & 1 & 2 & 113 & 37 & 37 & $I$ \\
\hline 2 & $0-5$ & 285 & 271 & 1 & 2 & 3.2 & 舟事 & 46 & 1 \\
\hline 2 & $0-4$ & 91 & 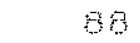 & 1 & 2 & 153 & 35 & 35 & I \\
\hline 2 & -3 & 962 & 352 & 1. & 2 & $2-13$ & 35 & 34 & t \\
\hline 2 & -2 & 606 & 575 & 2 & 2 & $2-12$ & 36 & 36 & 1 \\
\hline 2 & -1 & 372 & 349 & 2 & 2 & $2-11$ & 38 & 37 & 1 \\
\hline 2 & 0 & 406 & 401 & 2 & 2 & $2-10$ & 74 & 72 & 1 \\
\hline 2 & 1 & 94 & 96 & 1 & 2 & $2-9$ & 28 & 47 & 1 \\
\hline 2 & 2 & 133 & 130 & 1 & 2 & $2-7$ & 76 & 74 & $I$ \\
\hline 2 & 0 & 134 & 129 & 1 & 2 & $2-5$ & 98 & 96 & 1 \\
\hline 2 & 4 & 155 & 340 & 1 & 2 & $2-5$ & 92 & 93 & 1 \\
\hline 2 & 0 & 72 & 68 & 1 & 2 & $2-4$ & 216 & 206 & I \\
\hline 2 & 0 & 223 & 215 & $I$ & 2 & $2-3$ & 177 & 177 & 1 \\
\hline 2 & 0 & 56 & 53 & 1. & 2 & $2-2$ & 120 & 126 & 1 \\
\hline 2 & 8 & 40 & 40 & $\mathrm{i}$ & 2 & $2-1$ & 186 & 280 & 1 \\
\hline 2 & 0 & 73 & 75 & 1 & 2 & 20 & 168 & 175 & 1 \\
\hline 2 & 10 & 30 & 29 & 1 & 2 & a & 186 & 165 & 1 \\
\hline 2 & $0 \quad 11$ & 37 & 36 & I. & 2 & 2 & 189 & 196 & l. \\
\hline 2 & 12 & 1. & 10 & $\underline{z}$ & 2 & 3 & 64 & 61 & 1 \\
\hline 2 & 13 & is & 35 & 1 & 2 & 4 & 191 & 182 & 1 \\
\hline 2 & $1-13$ & 25 & 25 & 1 & 2 & 5 & 75 & 69 & 1. \\
\hline 2 & $1-12$ & 38 & 38 & 1 & 2 & 6 & 73 & 73 & 1. \\
\hline 2 & $1-11$ & 20 & 18 & 1 & 2 & 7 & 49 & 49 & 1 \\
\hline 2 & $1-10$ & 14 & I & I & 2 & 6 & 32 & 32 & I \\
\hline 2 & $1-9$ & o & 81 & $i$ & 2 & 9 & 72 & 71 & I \\
\hline 2 & -8 & 29 & 29 & 0 & 2 & $\approx 10$ & 71 & 73 & 1 \\
\hline 2 & -7 & 55 & 39 & 0 & 2 & $2 \quad 11$ & 49 & 40 & 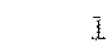 \\
\hline 2 & $-b$ & 31 & 29 & 0 & 2 & 212 & 52 & 52 & 1 \\
\hline 2 & -5 & {$[74$} & 364 & 1 & 2 & 219 & 34 & 36 & $I$ \\
\hline 2 & -4 & 193 & 130 & L & 2 & $3-13$ & 19 & 19 & 1 \\
\hline 2 & -3 & 922 & 331 & 1 & 2 & $9-12$ & 18 & 20 & $i$ \\
\hline 2 & -2 & 324 & 331 & I & 2 & $3-11$ & 39 & 39 & 1 \\
\hline 2 & -1 & 86 & 60 & 1 & 2 & -10 & 25 & 24 & 1 \\
\hline 2 & 0 & 164 & 159 & 1 & 2 & $3-9$ & 39 & 38 & 1 \\
\hline 2 & 1 & 256 & 255 & 1 & 2 & $5-8$ & 72 & 73 & 1 \\
\hline 2 & 2 & 295 & 297 & 1 & 2 & $3-7$ & 51 & 52 & \\
\hline
\end{tabular}




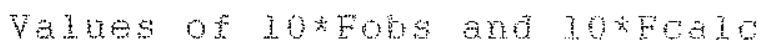

Page 7

\begin{tabular}{|c|c|c|c|c|c|c|c|c|c|c|}
\hline $\bar{H}$ & L & $70 \mathrm{ss}$ & poto & 519 & $\mathrm{H}$ & $\mathrm{E}$ & L & Fobs & Fondc & 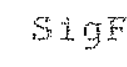 \\
\hline- & - & $-\cdots$ & $\cdots-\cdots$ & $\cdots \cdots$ & - & - & - & $\cdots-$ & $\ldots-\cdots$ & \\
\hline 2 & -6 & 16 & 14 & $\mathbf{I}$ & 2 & 5 & -7 & 4.4 & 44 & \\
\hline 2 & -5 & 10 & 39 & 1 & 2 & 5 & -6 & 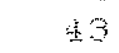 & 45 & . \\
\hline 2 & $3-4$ & 19 & $11 \mathrm{~B}_{2}$ & 1 & 2 & 5 & -5 & 36 & 37 & \\
\hline 2 & -3 & 48 & 49 & 3 & 2 & 5 & -4 & 91 & 94 & \\
\hline 2 & $3-2$ & 3.93 & 130 & $I$ & 2 & 5 & -3 & 37 & 36 & \\
\hline 2 & -1 & 105 & 103 & 1 & 2 & 5 & -2 & 124 & 123 & \\
\hline 2 & 0 & 57 & 61 & 1 & 2 & 5 & -1 & 129 & 129 & \\
\hline 2 & 1 & 365 & 163 & 1 & 2 & 5 & 1 & 39 & 38 & \\
\hline 2 & 2 & 150 & 146 & $i$ & 2 & 5 & 2 & 43 & 45 & \\
\hline 2 & 3 & 1.97 & 194 & 1 & 2 & 5 & 3 & 112 & 1.13 & \\
\hline 2 & 4 & 125 & 127 & $\underline{1}$ & 2 & 5 & 4 & 55 & 57 & . \\
\hline 2 & $E_{3}$ & 57 & 54 & $I$ & 2 & 5 & 5 & 103 & 106 & . \\
\hline 2 & 6 & 27 & 27 & 0 & 2 & 5 & 6 & 20 & 21 & . \\
\hline 2 & 7 & 189 & 187 & 1 & 2 & 5 & 7 & 67 & 66 & . \\
\hline 2 & 8 & 52 & 53 & 1 & 2 & 5 & 8 & 39 & 40 & 1 \\
\hline 2 & 9 & 23 & 23 & 1. & 2 & 5 & 10 & 27 & 29 & 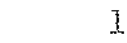 \\
\hline 2 & 310 & 96 & 100 & 1 & 2 & 5 & I] & 27 & 26 & I \\
\hline 2 & 311 & 22 & 19 & 3 & 2 & 5 & 12 & $\tilde{I}$ & 17 & 1 \\
\hline 2 & $3 \quad 3$ & 21 & 2. & 1 & 2 & & 1. 1 & 10 & II & 1 \\
\hline 2 & -1.1 & 29 & 29 & 1 & 2 & & 30 & 28 & 28 & 3 \\
\hline 2 & $4-10$ & 44 & 45 & 1 & 2 & 6 & -9 & 3 & 22 & I \\
\hline 2 & $4 \quad-9$ & 31 & 31 & 1 & 2 & 6 & -8 & 29 & 29 & 1 \\
\hline 2 & $\frac{28}{2}-8$ & 50 & 54 & 1 & 2 & 6 & -7 & 56 & 56 & 1 \\
\hline 2 & $\because-6$ & 8 & 13 & $i$ & 3 & 6 & -6 & 29 & 29 & 1 \\
\hline 2 & -5 & 24 & 25 & 0 & 2 & 6 & -5 & 59 & 58 & I. \\
\hline 2 & -4 & 63 & 69 & $i$ & 2 & 6 & -4 & 122 & 127 & 1 \\
\hline 2 & -3 & 22 & 21 & 0 & 2 & 6 & -3 & 60 & 59 & I \\
\hline 2 & -2 & 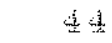 & 45 & 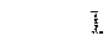 & 2 & 6 & -2 & 11 & 13 & 1 \\
\hline 2 & -1 & 102 & 104 & 1 & 2 & 6 & -1 & 22 & 22 & 1 \\
\hline 2 & 0 & 70 & 72 & I. & 2 & 6 & 0 & 39 & 38 & 1 \\
\hline 2 & $I$ & 50 & 47 & $\underline{i}$ & 2 & 6 & 1 & 31 & 33 & 0 \\
\hline 2 & 2 & 111 & 115 & 1 & 2 & 6 & 2 & 46 & 49 & 1 \\
\hline 2 & 3 & 94 & 92 & 1 & 2 & 6 & 3 & 39 & $4 I$ & I \\
\hline 2 & 4 & 38 & 40 & 0 & 2 & 6 & 委 & 38 & 38 & 1 \\
\hline 2 & 5 & 152 & 150 & 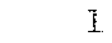 & 2 & 6 & 5 & 22 & 22 & 1 \\
\hline 2 & 6 & +4 & 43 & I. & 2 & 6 & 6 & 34 & 36 & 1 \\
\hline 2 & 7 & 124 & 120 & $\underline{1}$ & 2 & 6 & 8 & 19 & 19 & I \\
\hline 2 & 8 & 3 & 37 & 1 & 2 & 6 & 9 & 27 & 29 & 1 \\
\hline 2 & 9 & 18 & 19 & 1 & 2 & 6 & 10 & 17 & 14 & I \\
\hline 2 & 10 & 38 & 39 & 1 & 2 & 6 & 11 & 15 & 15 & 1 \\
\hline 2 & 31 & 13 & 13 & 1 & 2 & $7-$ & 10 & 14 & 10 & 1 \\
\hline 2 & 13 & 23 & 23 & I & 2 & 7 & -8 & 18 & 19 & 1 \\
\hline 2 & -9 & 12 & 12 & 1 & 2 & 7 & -7 & 23 & 24 & 1 \\
\hline 2 & -8 & 27 & 28 & 1 & $z$ & 7 & -6 & 46 & 6 & \\
\hline
\end{tabular}




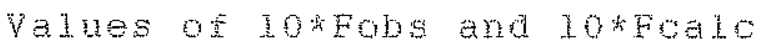

Page 8

\begin{tabular}{|c|c|c|c|c|c|c|c|c|c|c|}
\hline 垌 & $L_{i}$ & Fobs & FCalc & $S I g F$ & $H$ & K & $\mathrm{L}$ & Fobs & $F c a 1 c$ & SigF \\
\hline- & - & $\ldots-\cdots$ & $-\ldots \ldots-$ & $-\cdots$ & - & - & - & $\cdots-$ & $-\cdots-$ & $-\ldots-$ \\
\hline 7 & -55 & 17 & 16 & 1 & 2 & 10 & $I$ & 16 & 15 & $I$ \\
\hline 7 & -4 & 45 & 35 & $\mathrm{I}$ & 2 & 10 & 2 & 19 & 18 & I \\
\hline 7 & -3 & 43 & 43 & 1 & 3 & $0-$ & 13 & 23 & 23 & I \\
\hline 7 & -2 & 24 & 23 & 1 & 3 & $0-$ & 12 & 50 & 50 & I \\
\hline 7 & -1 & 58 & 63 & $I$ & 3 & $0-$ & 10 & 58 & 56 & I \\
\hline 7 & 0 & 30 & 33 & 1 & 3 & 0 & -9 & 36 & 88 & I \\
\hline 7 & 1 & 重壁 & 15 & $I$ & 3 & 0 & $-\theta$ & 52 & 53 & 1 \\
\hline 7 & 2 & 56 & 55 & 1 & 3 & 0 & -7 & 136 & 1.40 & 1 \\
\hline 7 & 3 & 21 & 22 & 1 & 3 & 0 & -6 & 22 & 21 & 0 \\
\hline 7 & 4 & 23 & 23 & I & 3 & 0 & -5 & 99 & 95 & 1 \\
\hline 7 & 5 & 19 & 17 & 3 & 3 & 0 & -4 & 271 & 264 & 1 \\
\hline 7 & 6 & 35 & $2 \pm$ & 1 & 3 & 0 & -3 & 316 & 307 & 1. \\
\hline 7 & 8 & 23 & 23 & I & 3 & 0 & -2 & 251 & 259 & I \\
\hline 7 & 9 & 33 & 31 & 1 & 3 & 0 & -1 & 169 & 171 & 1 \\
\hline 7 & 10 & 17 & 17 & $i$ & 3 & 0 & 0 & 61 & 172 & 1 \\
\hline 8 & -9 & 12 & 12 & 1. & 3 & 0 & $I$ & 75 & 75 & ]. \\
\hline 8 & -5 & 10 & 19 & 1 & 3 & 0 & 2 & 27 & 28 & 0 \\
\hline 8 & -7 & 22 & 20 & $\vec{i}$ & 3 & 0 & 3 & 109 & 102 & 1 \\
\hline 8 & -5 & 28 & 27 & $I$ & 3 & 0 & 4 & 56 & 55 & 1 \\
\hline 8 & -3 & 19 & 19 & 1 & 3 & 0 & 5 & 54 & 51 & 1. \\
\hline 8 & -2 & 46 & 47 & I & 3 & 0 & 5 & 192 & 178 & 1 \\
\hline 0 & $-I$ & 17 & 16 & 1 & 3 & 0 & 7 & 45 & 46 & 1 \\
\hline 8 & 0 & 49 & 48 & 1 & 3 & 0 & 8 & 86 & 87 & I \\
\hline 8 & 1 & 24 & 26 & 1. & 3 & 0 & 9 & 24 & 26 & 1 \\
\hline 8 & 2 & 12 & 12 & 1. & 3 & 0 & 10 & 42 & 41 & 1 \\
\hline 8 & 3 & 27 & 29 & 1 & 3 & 0 & 11 & 81 & 80 & 1 \\
\hline 8 & $\frac{d i}{2}$ & 50 & 29 & 1 & 3 & 0 & 12 & 28 & 30 & 1 \\
\hline 8 & 5 & 3.7 & 17 & $\underline{I}$ & 3 & $1-$ & 12 & 49 & 49 & 1 \\
\hline 8 & 8 & 23 & 23 & $\underline{I}$ & 3 & I- & 11 & 15 & 14 & I \\
\hline 9 & -7 & 17 & 15 & 1 & 3 & & 10 & 38 & 37 & 1 \\
\hline 9 & -6 & 27 & 36 & 1 & 3 & I & -9 & 97 & 88 & I \\
\hline 9 & -5 & 19 & 18 & 1 & 3 & I. & -8 & 72 & 73 & 1 \\
\hline 9 & $-\frac{4}{1}$ & $\mathrm{j}_{0}$ & 12 &. & 3 & I & -7 & 9 & 94 & 1 \\
\hline 9 & -3 & 11 & 12 & $\overrightarrow{1}$ & 3 & 1 & -6 & 59 & 58 & 1 \\
\hline 9 & -2 & 39 & 90 & 1 & 3 & 1 & -5 & 70 & 74 & 1 \\
\hline 9 & 0 & 1 & 16 & $i$ & 3 & I & -4 & 225 & 222 & I \\
\hline 9 & $I$ & 34 & 34 & 1 & 3 & 1 & -3 & 175 & 370 & I \\
\hline 9 & 2 & 15 & 16 & 1 & 3 & 1 & -2 & 69 & 69 & $I$ \\
\hline 9 & 4 & 39 & 16 & 1 & 3 & I & -1 & 249 & 242 & 1 \\
\hline 9 & 5 & 16 & 12 & 1 & 3 & 1 & 0 & 1.27 & 129 & 1 \\
\hline 9 & 6 & 30 & 26 & 爰 & 3 & 1 & I & 135 & 137 & 1 \\
\hline 9 & 7 & 25 & 23 & 1 & 3 & 1. & 2 & 85 & 86 & 1 \\
\hline 10 & -3 & 16 & 17 & 1 & 5 & I & 3 & 80 & 79 & I. \\
\hline 10 & -3 & 21 & 20 & 1 & 3 & I. & 每 & 355 & 345 & I. \\
\hline
\end{tabular}




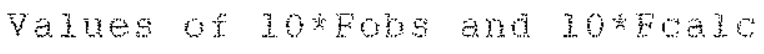

page 9

\begin{tabular}{|c|c|c|c|c|c|c|c|c|c|c|}
\hline I & E & $\mathrm{ECOS}$ & $\operatorname{Fec} \theta$ & 510 & $\mathrm{H}$ & $\mathrm{K}$ & $I_{4}$ & Fos & $F C a]$ & $\Leftrightarrow \mathrm{gl}$ \\
\hline - & - & --- & $--\rightarrow--$ & $\ldots-\cdots$ & - & - & - & $-\ldots$ & $-\ldots \ldots$ & $-\ldots-$ \\
\hline 3 & 5 & 99 & 97 & $I$ & 3 & 3 & -3 & 122 & 120 & $?$ \\
\hline 3 & 6 & 95 & 92 & $\underline{I}$ & 3 & 3 & -2 & 4 & 4 & 1. \\
\hline 3 & 7 & 69 & 87 & I & 3 & 3 & $-I$ & 31 & 30 & 0 \\
\hline 3 & 8 & $5 \frac{1}{7}$ & 55 & 1 & 3 & 3 & 0 & 86 & 98 & 1 \\
\hline 3 & 9 & 100 & 107 & 1 & 3 & 3 & $I$ & I. 1 & 114 & 1 \\
\hline 3 & 110 & 47 & 45 & $\mathrm{I}$ & 3 & 3 & 2 & 83 & 96 & 1 \\
\hline 3 & 131 & 43 & 40 & 1 & 7 & 3 & 3 & 128 & 131 & 1 \\
\hline 3 & $1 \quad 32$ & 37 & 37 & 1 & 3 & 3 & 4 & 2.7 & 45 & 1 \\
\hline 3 & 113 & 23 & 23 & I. & 3 & 3 & 5 & 139 & 138 & 1 \\
\hline 3 & $2-13$ & 14 & 15 & 1 & 3 & 3 & 6 & 49 & 50 & 1. \\
\hline 7 & $2-12$ & 25 & 24 & $I$ & 3 & 3 & 7 & 66 & 66 & 1 \\
\hline
\end{tabular}

